\title{
A GEOMETRICAL PROCEDURE FOR KILLING THE MIDDLE DIMENSIONAL HOMOLOGY GROUPS OF ALGEBRAIC HYPERSURFACES
}

\author{
A. LIBGOBER
}

\begin{abstract}
Explicit construction for decomposition of algebraic hypersurfaces into a connected sum of handles and a homological projective space is discussed. Also a connection is provided between Levine's results about the Arf invariant in the theory of knots and the computation of Arf invariant of hypersurfaces by S. Morita and J. Wood.
\end{abstract}

Morita [1] and Wood [2] have recently proved the following theorem:

THEOREM 1. Let $V_{n}^{d}$ be a nonsingular algebraic hypersurface of an odd dimension $n$ and of degree $d$ in an $(n+1)$-dimensional complex projective space. Then there are two cases:

(i) If $d \neq \pm 3$ (8) or $n=1,3,7$, then $V_{n}^{d}$ is decomposable into a connected sum of copies of $S^{n} \times S^{n}$, and a differentiable manifold $M_{n}^{d}$ with middle Betti number zero.

(ii) If $d \equiv \pm 3$ (8) and $n \neq 1,3,7$, then $V_{n}^{d}$ is decomposable into a connected sum of copies of $S^{n} \times S^{n}$ and a differentiable manifold $M_{n}^{d}$ with middle Betti number 2 and Kervaire invariant 1.

In this paper we describe an explicit geometrical construction for getting such a decomposition, from which Theorem 1 will follow. Our theorem however will not include the case $n=4 k+1, d \equiv 4,6(\bmod 8)$.

In subsequent work we shall study in more detail the topological structure of $M_{n}^{d}$ starting with the description of it obtained below, and also use the degeneration discussed here to get some information about the topology of even dimensional projective hypersurfaces.

We study a special hypersurface $V_{n}^{d}(c)$ which is a projective closure of an affine hypersurface defined by the equation

$$
P_{n}\left(Z_{0} \cdots Z_{n}\right)=Z_{0}^{d}+Z_{1}^{d-1}+Z_{1} Z_{2}^{d-1}+\cdots+Z_{n-1} Z_{n}^{d-1}=C .
$$

In the following theorem we discuss some properties of this hypersurface.

THEOREM 2. (i) The hypersurface $V_{n}^{d}(c)$ is a nonsingular projective variety for $c \neq 0$, and has a single isolated singularity for $c=0$ at the point $Z_{0}=Z_{1}$ $=\cdots=Z_{n}=0$.

Received by the editors April 26, 1976 and, in revised form, September 30, 1976. AMS (MOS) subject classifications (1970). Primary 14M99, 57A15, 57D55. 
(ii) If $C$ is sufficiently close to zero then the intersection of $V_{n}^{d}(c)$ with a disk of a small radius $\varepsilon$, centered at the origin, is an $(n-1)$-connected $(2 n)$-manifold $F_{n}^{d}$ and its $n$-dimensional Betti number $b_{n}$ is given by

$$
b_{n}=d^{-1}\left[(d-1)^{n+2}-(d-1)\right] \text {. }
$$

(iii) The characteristic polynomial $\Delta_{n}(t)$ of the monodromy of the isolated singularity of $V_{n}^{d}$ can be computed by the recursive equation

$$
\begin{aligned}
\Delta_{n+2}(t) & =\Delta_{n}(t) \frac{t^{d(d-1)^{n+2}}-1}{t^{(d-1)^{n+2}}-1} \frac{t^{(d-1)^{n+1}}-1}{t^{d(d-1)^{n+1}}-1}, \\
\Delta_{1}(t) & =\frac{t^{d(d-1)}-1}{t^{d}-1} \frac{(t-1)}{t^{(d-1)}-1} .
\end{aligned}
$$

(iv) If $n$ is odd then

$$
\begin{aligned}
\Delta_{n}(1) & =1, \\
\Delta_{n}(-1) & =d \text { for } d \text { odd }, \\
\Delta_{n}(-1) & =(d-1)^{(n+1) / 2} \text { for } d \text { even. }
\end{aligned}
$$

Now let $T_{2 n}$ be a $2 n$-dimensional manifold which is a plumbing of two copies of a tangent bundle of the sphere $S^{n}$. Since $\Delta_{n}(1)=1$, it is known that the boundary of $T_{2 n}$ is homeomorphic to $S^{2 n-1}$. Denote by $D^{2 n}$ the disk of dimension $2 n$. Then we have the following theorem.

THEOREM 3. Define the manifold $M_{n}^{d}$ as a gluing of $V_{n}^{d}(C)-F_{n}^{d}$ and $D^{2 n}$ when $d \neq \pm 3(8)$, or $d \neq 4,6 \bmod (8)$ and $n=4 k+1$, or $n=1,3,7$. Otherwise define $M_{n}^{d}$ as a gluing of $V_{n}^{d}(C)-F_{n}^{d}$ and $T_{2 n}$. In the first case $M_{n}^{d}$ is a closed manifold with vanishing middle homology group. In the second case the n-dimensional Betti number of $M_{n}^{d}$ is 2 and its Kervaire invariant is 1, except in the case when $d \equiv 4,6(\bmod 8)$ and $n=4 k+1$. In both cases the hypersurface $V_{n}^{d}(C)$ is diffeomorphic to a connected sum of $M_{n}^{d}$ and copies of $S^{n} \times S^{n}$.

In the case $d \neq 4,6(\bmod 8)$ and $n=4 k+1$ all assertions of Theorem 1 are evidently contained in Theorem 3 .

Proof of Theorem 3. From the results of Levine [3] it follows that $F_{n}^{d}$ has Kervaire invariant one if $\Delta(-1) \equiv \pm 3(8)$, and Kervaire invariant zero if $\Delta(-1) \equiv \pm 1(8)$. Hence we can derive the value of the Kervaire invariant of $F_{n}^{d}$ by (iv) of Theorem 2 . Since the boundary of $F_{n}^{d}$ is a homotopy sphere, Wall's results [4] show the diffeomorphism type of $F_{n}^{d}$ is determined by the Betti number $b_{n}$ and the Kervaire invariant. Hence for $d \not \pm 3$ (8) or $d \neq 4$, 6 , and $n=4 k+1$ or $n=1,3,7, F_{n}^{d}$ is diffeomorphic to a connected sum of copies of $S^{n} \times S^{n}$ with removed disk, otherwise $F_{n}^{d}$ is a connected sum of $T_{2 n}$ and copies of $S^{n} \times S^{n}$. The assertion about the Betti number of $M_{n}^{d}$ follows from the well-known fact that the Betti number of a nonsingular hypersurface of odd dimension $n$ and degree $d$ is equal to $d^{-1}\left[(d-1)^{n+2}-(d-1)\right]$ (i.e., to the Betti number of $\left.F_{n}^{d}\right)$. This completes the proof of Theorem 3 . 
Proof of Theorem 2. Assertion (i) follows by a standard computation. Next recall [5] that the polynomial $f\left(Z_{0} \cdots Z_{n}\right)$ is weighted homogeneous of type $\left(W_{0} \cdots W_{n}\right)$ if it can be expressed as a linear combination of monomials $Z_{0}^{i_{0}} \cdots Z_{n}^{i_{n}}$ for which

$$
i_{0} / W_{0}+i_{1} / W_{1}+\cdots+i_{n} / W_{n}=1 \text {. }
$$

We claim that polynomial $P_{n}\left(Z_{0} \cdots Z_{n}\right)$ is weighted homogeneous of the type

$$
\left(d, d-1, \ldots, \frac{d(d-1)^{i}}{(d-1)^{i}+(-1)^{i-1}}, \ldots, \frac{d(d-1)^{n}}{(d-1)^{n}+(-1)^{n-1}}\right) .
$$

This follows since (1) can be checked directly for every monomial of the polynomial $P\left(Z_{0} \cdots Z_{n}\right)$.

To complete the proof of Theorem 2 we need the results of Milnor and Orlik [5] which we describe now.

To each polynomial of one variable $\left(t-\alpha_{1}\right), \ldots,\left(t-\alpha_{n}\right)$ assign the element $\left\langle\alpha_{1}\right\rangle+\cdots+\left\langle\alpha_{n}\right\rangle$ of the group ring $Z\left[\mathbf{C}^{*}\right]$ of the group $\mathbf{C}^{*}$. We denote this element by

$$
\operatorname{div}\left(\left(t-\alpha_{1}\right) \cdots\left(t-\alpha_{n}\right)\right)
$$

and let $\Lambda_{n}$ denote $\operatorname{div}\left(t^{n}-1\right)$. Milnor and Orlik proved

THEOREM 4 [5]. Let $f\left(Z_{0} \cdots Z_{n}\right)$ be a weighted homogeneous polynomial of type $\left(W_{0} \cdots W_{n}\right)$ having an isolated singularity at the point $Z_{0}=\cdots=Z_{n}$ $=0$. Then for $C$ sufficiently close to zero the intersection of the hypersurface $f\left(Z_{0} \cdots Z_{n}\right)=C$ with a disk of small radius $\varepsilon$ centered at the origin, is an $(n-1)$-connected $2 n$-manifold and

(a) the rank of the $n$-dimensional homology group of this intersection equals $\left(W_{0}-1\right) \cdots\left(W_{n}-1\right)$

(b) if $\Delta(t)$ is the characteristic polynomial of monodromy of the isolated singularity of $f\left(Z_{0} \cdots Z_{n}\right)=0$ then

$$
\operatorname{div} \Delta_{n}(t)=\left(v_{0}^{-1} \Lambda_{u_{0}}-1\right) \cdots\left(v_{n} \Lambda_{u_{n}}-1\right)
$$

where the weights are expressed in the form $w_{i}=u_{i} / v_{i}(i=0, \ldots, n)$ with $u_{i} v_{i}$-mutually prime integer numbers.

We are ready to show (ii). We use the values of the weight $W_{0} \cdots W_{n}$ which were found above, and then from (a) of Theorem 4 it follows that the expression for the rank of $H_{n}\left(F_{n}^{d}, Z\right)$ is

$$
\begin{aligned}
\mu & =\left(W_{0}-1\right) \cdots\left(W_{n}-1\right)=(d-1) \prod_{i=1}^{n}\left[\frac{d(d-1)^{i}}{(d-1)^{i}+(-1)^{i-1}}-1\right) \\
& =(d-1) \prod_{i=1}^{n} \frac{(d-1)^{i+1}+(-1)^{i}}{(d-1)^{i}+(-1)^{i-1}} \\
& =d^{-1}\left[(d-1)^{n+2}-(d-1)\right] .
\end{aligned}
$$


(iii) We prove now that

$$
\operatorname{div} \Delta_{n}(t)=\sum_{i=0}^{n}(-1)^{n-i} \Lambda_{d(d-1)^{i}}+\sum_{i=0}^{n}(-1)^{n-i-1} \Lambda_{(d-1)^{i}}
$$

The proof of (2) is based on the following identities [5]:

$$
\begin{aligned}
\Lambda_{(d-1)^{n+1}} \Lambda_{d(d-1)^{i}} & =(d-1)^{i} \Lambda_{d(d-1)^{n+1}}, \\
\Lambda_{(d-1)^{n+1}} \Lambda_{(d-1)^{i}} & =(d-1)^{i} \Lambda_{(d-1)^{n+1}} .
\end{aligned}
$$

The inductive step follows from the next computation:

$$
\begin{aligned}
& \operatorname{div} \Lambda_{n+1}=\operatorname{div} \Lambda_{n} \cdot\left(\frac{d}{(d-1)^{n+1}+(-1)^{n}} \Lambda_{(d-1)^{n+1}}-1\right) \\
& =\left(\sum_{i=0}^{n}(-1)^{n-i} \Lambda_{d(d-1)^{i}}+\sum_{i=0}^{n}(-1)^{n-i-1} \Lambda_{(d-1)^{i}}\right) \\
& \times\left(\frac{d}{(d-1)^{n+1}+(-1)^{n}} \Lambda_{(d-1)^{n+1}-1}\right) \\
& =\sum_{i=0}^{n}(-1)^{n-i-1} \Lambda_{d(d-1)^{i}}+\sum_{i=0}^{n}(-1)^{n-i} \Lambda_{(d-1)^{i}} \\
& +\left[\frac{d}{(d-1)^{n+1}+(-1)^{n}} \sum_{i=0}^{n}(-1)^{n-i}(d-1)^{i}\right] \Lambda_{d(d-1)^{n+1}} \\
& +\left[\frac{d}{(d-1)^{n+1}+(-1)^{n}} \sum_{i=0}^{n}(-1)^{n-i}(d-1)^{i}\right] \Lambda_{d(d-1)^{n+1}} \\
& =\sum_{i=0}^{n}(-1)^{n-i+1} \Lambda_{d(d-1)^{i}} \\
& +\sum_{i=0}^{n}(-1)^{n-i} \Lambda_{(d-1)^{i}}+(-1)^{n+1} \Lambda_{d(d-1)^{n+1}}+(-1)^{i} \Lambda_{(d-1)^{n+1}} \\
& =\sum_{i=0}^{n+1}(-1)^{n+1-i} \Lambda_{d(d-1)^{i}}+\sum_{i=0}^{n+1}(-1)^{n-i} \Lambda_{(d-1)^{i}} .
\end{aligned}
$$

From (2) it follows:

$$
\Delta_{n+1}(t)=\Delta_{n}^{-1}\left(t^{d(d-1)^{n+1}}-1\right)\left(t^{(d-1)^{n+1}}-1\right)^{-1} \text {. }
$$

The recursive equation mentioned in Theorem 2 is implied by the last formula. The assertion about $\Delta_{1}$ follows immediately from Theorem 4 .

(iv) It can be seen from the recursive equation in (iii) that

$$
\begin{aligned}
\Delta_{n}(1) & =\Delta_{n+2}(1), & & \\
\Delta_{n}(-1) & =\Delta_{n+2}(-1) & & \text { for } d \text { odd, and } \\
(d-1) \Delta_{n}(-1) & =\Delta_{n+2}(-1) & & \text { for } d \text { even. }
\end{aligned}
$$


The value $\Delta_{1}(1)$ equals 1 and $\Delta_{1}(-1)$ equals $d$ for $d$ odd and $(d-1)$ for $d$ even.

Thus all assertions of Theorem 2 are proved.

I am grateful to the referee for his very interesting and important remarks. I also wish to thank Professor Moishezon for his help and advice.

\section{REFERENCES}

1. S. Morita, The Kervaire invariant of hypersurfaces in complex projective spaces, Comment. Math. Helv. 50 (1975), 403-419. MR 52 \#6756.

2. J. Wood, Removing handles from nonsingular algebraic hypersurfaces in $C P_{n+1}$, Invent. Math. 31 (1975), 1-3.

3. J. Levine, Polynomial invariant of knots of codimension two, Ann. of Math. (2) 84 (1966), 537-554.

4. C. T. C. Wall, Classification of $(n-1)$-connected $2 n$-manifolds, Ann. of Math. (2) 75 (1962), 163-198. MR 26 \#3071.

5. J. Milnor and P. Orlik, Isolated singularity defined by weighted homogeneous polynomials, Topology 9 (1970), 385-393. MR 45 \#2757.

Department of Mathematics, Tel-Aviv University, Tel-Aviv, IsRael 